\title{
A Class of Non-Kekulé Molecules with Low Excitation Energies
}

\author{
Fritz Dietz $^{\mathrm{a}}$, Nedko Drebov ${ }^{\mathrm{b}}$, and Nikolai Tyutyulkov ${ }^{\mathrm{a}, \mathrm{b}}$ \\ ${ }^{a}$ Universität Leipzig, Wilhelm-Ostwald-Institut für Physikalische und Theoretische Chemie, \\ Johannisallee 29, D-04103 Leipzig, Germany \\ $\mathrm{b}$ University of Sofia, Faculty of Chemistry, Department of Physical Chemistry, 1 J. Bourchier blvd, \\ BG-1126, Sofia, Bulgaria
}

Reprint requests to Prof. F. Dietz. Fax: +49-341-36399. E-mail: dietz@ chemie.uni-leipzig.de

Z. Naturforsch. 2007, 62b, 1433 - 1436; received June 25, 2007

Dedicated to Professor Dr. Jürgen Fabian on the occasion of his $70^{\text {th }}$ anniversary

\begin{abstract}
A class of non-Kekulé molecular systems with a new structural principle and low excitation energies or with a triplet ground state was investigated theoretically. The systems consist of a non-Kekulé monoradical, possessing a non-bonding molecular orbital linked in a specific way to another monoradical.
\end{abstract}

Key words: Non-Kekulé Molecules, Triplet Diradicals, Molecular Systems with Low Excitation Energies

\section{Introduction}

In this communication we consider a class of nonKekulé molecules with a new structural principle and with (very) low excitation energies. The structural principle and the peculiarity of the investigated systems are described in detail in the following section.

The investigations presented in the paper are an extension of the studies in lit. [1], in which the same structural principle determines the very low excitation energy of a group of polymethine mono-radicals.

\section{Structural Principle of the Investigated Molecules}

$\pi$ Systems with NBMOs determined by the molecular topology

There are two classes of $\pi$ systems with nonbonding molecular orbitals (NBMO) determined by the molecular topology: homo-nuclear alternant systems and quasi-alternant systems, respectively.

\section{Homonuclear alternant radicals}

The presence of NBMOs in alternant non-classical (non-Kekulé) radicals and polyradicals follows from the Coulson-Rushbrooke-Longuet-Higgins (CRLH) theorem $[2,3]$. According to this theorem, a homonuclear alternant $\pi$-electron system (hydrocarbon) has at least

$$
N=S^{*}-U^{\circ}
$$

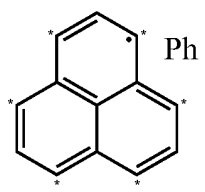<smiles></smiles>

$7^{*}-6=1 \mathrm{NBMO} \quad 2 \times 7^{*}-13=1 \mathrm{NBMO}$ Scheme 1 .

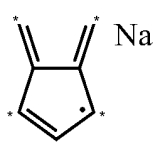

$2 \times 4 *-7=1 \mathrm{NBMO}$
NBMOs, where $\left\{S^{*}\right\}$ and $\left\{U^{\circ}\right\}$ are the number of the starred and un-starred $\pi$ centers, respectively. In such systems, for which the CRLH theorem is valid, every un-starred $C^{\circ}$ atom is connected with one or two starred $C^{*}$ atoms and vice versa. An example is the phenalenyl radical $(\mathrm{Ph})$ shown in Scheme 1 (here and below only one resonance formula is depicted).

\section{Quasi-alternant radicals}

The application of the CRLH theorem is limited to homonuclear alternant systems, i.e., alternant hydrocarbons. The theorem has been extended (ECRLH) $[4,5]$. Radicals for which the ECRLH theorem is valid are designated as quasi-alternant non-classical (nonKekulé) (QANC) radicals [6].

Within the CRLH theorem one always considers two subsets of homonuclear non-bonded $\pi$ centers: the subset of starred $\left\{S^{*}\right\}$ and the subset of un-starred $\left\{U^{\circ}\right\}$ ones. According to the ECRLH theorem [4,5] only one subset of non-bonded (disjoint) $\pi$ centers 


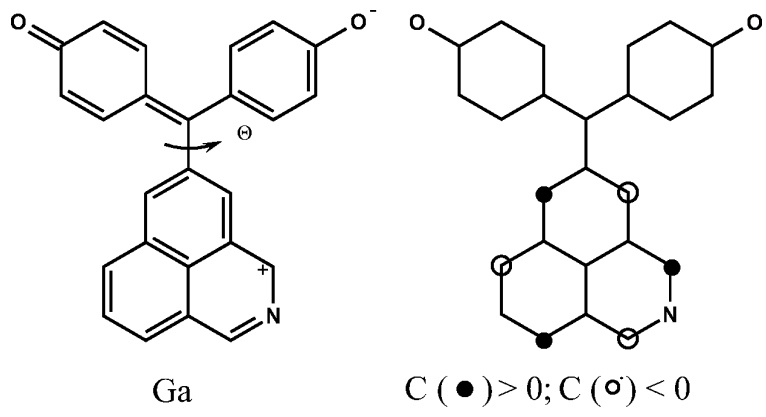

Fig. 1. Localization of the NBMO within the Az fragment of the $\mathrm{Az}^{\bullet}-\mathrm{Ga}^{\bullet}$ system.
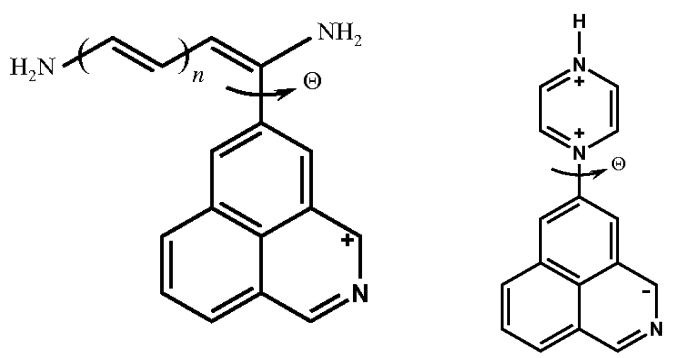

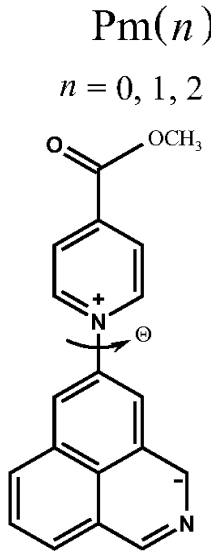

Ko

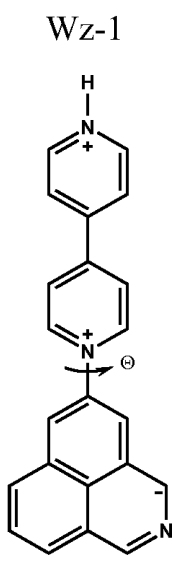

$\mathrm{Wz}-2$
Fig. 2. Investigated molecules. The Az radical is linked with polymethine radicals with an even number of methine groups, $\operatorname{Pm}(n)$, a Kosower-type radical, Ko, and Weitz-type radicals, Wz-1(2). The molecule Ga with the galvinoxyl radical is shown in Fig. 1.

is responsible for the appearance of NBMOs. A system with $M \pi$ centers and a maximum set of starred homonuclear non-bonded (disjoint) $\pi$ centers $\left\{S^{*}\right\}$ must have at least

$$
N=2 S^{*}-M
$$

NBMOs.
The NBMOs are present even if the $\pi$ system is non-alternant and if the $\pi$ centers belonging to the non-starred subset $\left\{U^{\circ}\right\}$ are heteroatomic or nonalternant systems. Typical examples are the stable 2azaphenalenyl radical (Az), which has been synthesized and characterized by Rubin et al. [7], and the non-alternant radical $(\mathrm{Na})$ (see Scheme 1).

The following corollary rooted in the ECRLH theorem (for a proof, see [5] and [1]) determines the structural principle of the investigated non-Kekule molecules.

Let us consider a $\pi$ system consisting of a radical subunit $\mathrm{R}^{\bullet}$, for which the CRLH or ECRLH theorem is valid, i. e., $\mathrm{R}^{\bullet}$ has one NBMO, linked with a monoradical $\mathrm{M}$ (the radical $\mathrm{M}^{\bullet}$ has no NBMO):

$$
\mathrm{R}^{\bullet}-\mathrm{M}^{\bullet}
$$

If the $\pi$ center $r$ of $\mathrm{R}^{\bullet}$ which is connected with a starred $\pi$ center $s$ of $\mathrm{M}^{\bullet}$ belongs to the set of the non-starred atoms $\left\{U^{\circ}\right\}$ the MO coefficient $C^{\circ}{ }_{\mathrm{r}}=0$, then all the NBMO coefficients in the fragment (radical) $\mathrm{M}^{\bullet}$ are zero, i.e., the NBMO coefficients are non-zero only for the starred $\pi$ centers in $\mathrm{R}^{\bullet}$, and the NBMO is strictly localized within the subunit (radical) $\mathrm{R}^{\bullet}$. The localization of the NBMO within the $\mathrm{Az}$ fragment of a $\mathrm{R}^{\bullet}-\mathrm{M}^{\bullet}$ system which consists of Coppinger's radical (galvinoxyl) [8] and the Az radical is illustrated in Fig. 1.

\section{Objects of Investigations}

The investigated systems are shown in Fig. 2. In all systems the radical subunit $\mathrm{R}^{\bullet}$ is the $\mathrm{Az}$ radical connected with a Kosower radical [9] Ko, a Weitztype radical [10 - 12] Wz-1(2), or polymethine radicals [13-15] (with an even number of methine groups between the terminal groups) $\operatorname{Pm}(n)$.

\section{Computational Methods}

Numerical results were obtained with the semiempirical AM1 method [16] using the MOPAC 97 program package. Numerical results were obtained also with AM1/CIS $(m, n)$, AM1/CISD $(m, n)$ and AM1/CAS $(m, n)$ methods using an upgraded version of the MOPAC program [17]. $m$ and $n$ are the numbers of the MOs determining the active CI space. For more detailed studies of the ground state multiplicity of the Wz-2 system, $a b$ initio and DFT methods were also performed (GAUSSIAN 03 [18]). 
Table 1. Calculated values [AM1/CAS $(16,16)]$ of the longest-wavelength singlet-singlet, $\Delta E_{\mathrm{S}}(1,2)$, and singlettriplet transitions, $\Delta E_{\mathrm{T}}(1,2)$, for the investigated molecules (Figs. 1 and 2). $f_{1(2)}$ are the corresponding oscillator strengths. All entries are in eV. $\Theta$ represents the AM1 optimized dihedral angles in degrees.

\begin{tabular}{cccccrrr}
\hline Molecule & $\Theta$ & $\Delta E_{\mathrm{S}}(1)$ & $f_{1} \times 10^{3}$ & $\Delta E_{\mathrm{S}}(2)$ & $f_{2} \times 10^{3}$ & $\Delta E_{\mathrm{T}}(1)$ & $\Delta E_{\mathrm{T}}(2)$ \\
\hline $\operatorname{Pm}(0)$ & 45.0 & 1.28 & 8 & 2.98 & 25 & 1.16 & 2.40 \\
$\operatorname{Pm}(1)$ & 44.7 & 0.75 & 2 & 2.68 & 100 & 0.69 & 2.14 \\
$\operatorname{Pm}(2)$ & 44.7 & 0.69 & 2 & 2.54 & 7 & 0.65 & 2.06 \\
Wz-1 & 53.8 & 0.92 & 6 & 2.77 & 26 & 0.74 & 2.28 \\
Wz-2 & 26.7 & 0.27 & 2 & 2.10 & 1 & -0.14 & 1.85 \\
Ko & 41.3 & 0.12 & 1 & - & - & -0.12 & 1.03 \\
Ga & 45.4 & 1.29 & 186 & 1.59 & 5 & 0.03 & 1.42 \\
\hline
\end{tabular}

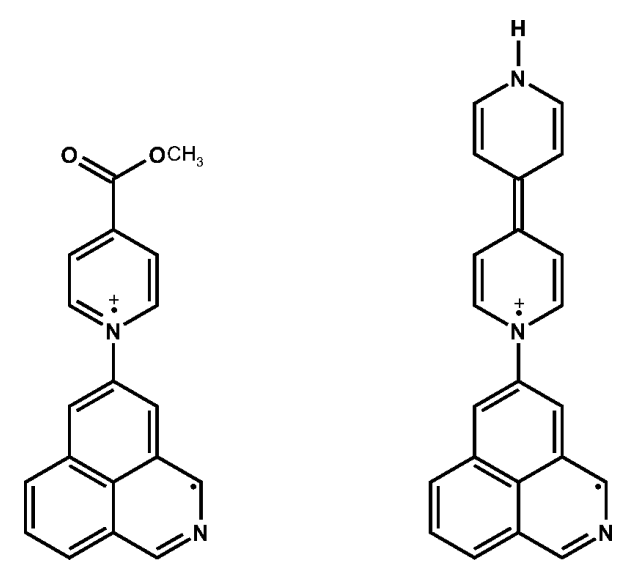

Scheme 2.

\section{Numerical Results and Discussion}

\section{Transition energies}

In Table 1 are given the calculated values of the longest-wavelength singlet-singlet and singlet-triplet transitions for the investigated molecules. The results were obtained by means of the AM1/CAS $(16,16)$ method using geometries optimized with the AM1 method.

In all cases the energies of the first singlet-singlet transitions are $\Delta E_{\mathrm{S}}(1)<1 \mathrm{eV}$ (with the exception of the systems $\operatorname{Pm}(0)$ and $\mathrm{Ga}$ ). Also the energies of the first singlet-triplet transitions are $\Delta E_{\mathrm{T}}(1)<1 \mathrm{eV}$.

The negative values of the first singlet-triplet transition of the Wz-2 and Ko systems indicate triplet states, i. e. the ground state corresponds to a triplet diradical. In Scheme 2 only one resonance formula is depicted for these systems.

The calculated values of the transition energies $\Delta E_{\mathrm{S}}(1)$ and $\Delta E_{\mathrm{T}}(1)$ of the $\operatorname{Pm}(0)$ molecule with different methods and with different widths of the configuration interaction (CI) are shown in Table 2. The
Table 2. Calculated values of the longest-wavelength singletsinglet and singlet-triplet transition energies $(\mathrm{eV})$ of the $\mathrm{Pm}(0)$ molecule with different methods and with different widths of the configuration interaction (see Section 4).

\begin{tabular}{lccc}
\hline Method & $\Delta E_{\mathrm{S}}(1)(\mathrm{eV})$ & $f \times 10^{3}$ & $\Delta E_{\mathrm{T}}(1)(\mathrm{eV})$ \\
\hline CIS $(8,8)$ & 1.57 & 23 & 1.28 \\
CIS $(14,14)$ & 1.54 & 17 & 1.23 \\
CIS $(20,20)$ & 1.53 & 17 & 1.18 \\
CISD $(8,8)$ & 1.41 & 13 & 1.57 \\
CISD $(14,14)$ & 1.55 & 10 & 1.54 \\
CISD $(20,20)$ & 1.51 & 10 & 1.39 \\
CAS $(8,8)$ & 1.20 & 11 & 1.04 \\
CAS $(14,14)$ & 1.28 & 8 & 1.17 \\
CAS $(20,20)$ & 1.33 & 9 & 1.21 \\
\hline
\end{tabular}

Table 3. Calculated energy differences, $\Delta E_{\mathrm{S}}(1)$ and $\Delta E_{\mathrm{T}}(1)$ (in $\mathrm{eV}$ ), obtained with semi-empirical post-HF and $a b$ initio methods, for molecule Wz-2.

\begin{tabular}{lll}
\hline Method & $\Delta E_{\mathrm{S}}(1)(\mathrm{eV})$ & $\Delta E_{\mathrm{T}}(1)(\mathrm{eV})$ \\
\hline AM1/CAS(10,10) & 0.29 & -0.14 \\
AM1/CAS(16,16) & 0.27 & -0.14 \\
AM1/CAS(20,20) & 0.36 & -0.11 \\
CASSCF(4,3)/3-21G* & 0.38 & -0.06 \\
B3LYP/3-21G* & 0.61 & -0.02 \\
B3LYP/6-31G** & 0.60 & -0.02 \\
\hline
\end{tabular}

results were obtained using the geometries optimized also with the AM1 method. The results in Table 2 show that the extension of the configuration interaction does not change the numerical results substantially. This result is valid also for the other investigated systems.

Table 3 presents the calculated energy differences $\Delta E_{\mathrm{S}}(1)$ and $\Delta E_{\mathrm{T}}(1)$ (in $\mathrm{eV}$ ) for molecule Wz-2 using $a b$ initio and DFT methods. The numerical results are a quantitative illustration of the important qualitative result, the triplet ground state of the $\mathrm{Wz}-2$ system. The results of the Ko system are similar.

\section{Conclusions and Outlook}

A class of non-Kekule molecular systems with a new structural principle and low exitation energies was investigated theoretically. The systems consist of a non-Kekulé monoradical, $\mathrm{R}^{\bullet}$, possessing a nonbonding molecular orbital (NBMO), and are connected with another monoradical, $\mathrm{M}^{\bullet}$, without $\mathrm{NBMO}(\mathrm{s})$. Here, only systems (molecules) $\mathrm{Az}^{\bullet}-\mathrm{M}^{\bullet}$ with one example of a radical subunit $\mathrm{R}^{\bullet}$, namely the 2-azaphenalenyl radical, Az, have been studied. The multiformity of the stable radicals of types $\mathrm{R}^{\bullet}$ and $\mathrm{M}^{\bullet}$ determines the expediency of the theoretical and experimental investigations of this group of molecular systems. 
The investigated model compounds are characterized by very low excitation energies with longestwavelength absorptions in the near infrared region $[11,19]$. The molecular species are candidates of building blocks of one-dimensional (1-D) stacks with intermolecular $\pi$-electron systems of conjugation with non-conventional physical properties. 1-D stacks with ferromagnetically coupled electrons within the half-filled band or with a metallic ground state can result depending on the multiplicity of the molecular building blocks [20-22].
[1] N. Tyutyulkov, N. Drebov, A. Staykov, A. Tadjer, Int. J. Quantum Chemistry 2007, 107, 1396.

[2] C. A. Coulson, S. Rushbrooke, Proc. Cambridge Phil. Mag. 1940, 36, 193.

[3] C. A. Coulson, H. C. Longuet-Higgins, Proc. Roy. Soc. London 1947, A191, 39; ibid. 1947, A192, 16; ibid. 1948, A193, 447; ibid. 1948, A195, 188.

[4] N. Tyutyulkov, O. E. Polansky, Chem. Phys. Lett. 1987, 139, 281.

[5] S. Karabunarliev, N. Tyutyulkov, Theoret. Chim. Acta 1989, 76, 65.

[6] N. Tyutyulkov, S. Karabunarliev, C. Ivanov, Mol. Cryst. Liq. Cryst. 1989, 176, 139.

[7] S. Zheng, J. Lan, S. I. Khan, Y. Rubin, J. Am. Chem. Soc. 2003, 125, 5786.

[8] G. M. Coppinger, J. Am. Chem. Soc. 1957, 79, 502.

[9] J. Hermolin, M. Levin, E. M. Kosower, J. Am. Chem. Soc. 1981, 103, 4808; J. Hermolin, M. Levin, Y. Ikegami, M. Sawayanangl, E. M. Kosower, J. Am. Chem. Soc. 1981, 103, 4795.

[10] E. Weitz, K. Fischer, Angew. Chem. 1925, 38, 1110.

[11] J. Fabian, H. Hartmann, Light Absorption of Organic Colorants, Springer, Berlin, 1980.

[12] N. Tyutyulkov, J. Fabian, A. Mehlhorn, F. Dietz, A. Tadjer, Polymethine Dyes - Structure and Properties, St. Kliment Ohridski University Press, Sofia, 1991.

[13] W. König, J. Prakt. Chem. 1926, 112, 1.

[14] S. Dähne, Z. Chem. 1965, 5, 441.

[15] S. Hünig, Liebigs Ann. Chem. 1964, 676, 32.

[16] J. J. J. Stewart, Mopac: A General Molecular Orbital Package (version 7.2), Quantum Chemistry Program Exchange, 1995.
[17] S. Karabunarliev, M. Baumgarten, K. Müllen, J. Phys. Chem. 1998, A 102, 7029.

[18] M. J. Frisch, G. W. Trucks, H. B. Schlegel, G. E. Scuseria, M. A. Robb, J. R. Cheeseman, J. A. Montgomery, Jr., T. Vreven, K. N. Kudin, J.C. Burant, J. M. Millam, S. S. Iyengar, J. Tomasi, V. Barone, B. Mennucci, M. Cossi, G. Scalmani, N. Rega, G. A. Petersson, H. Nakatsuji, M. Hada, M. Ehara, K. Toyota, R. Fukuda, J. Hasegawa, M. Ishida, T. Nakajima, Y. Honda, O. Kitao, H. Nakai, M. Klene, X. Li, J.E. Knox, H.P. Hratchian, J.B. Cross, V. Bakken, C. Adamo, J. Jaramillo, R. Gomperts, R. E. Stratmann, O. Yazyev, A. J. Austin, R. Cammi, C. Pomelli, J.W. Ochterski, P. Y. Ayala, K. Morokuma, G. A. Voth, P. Salvador, J. J. Dannenberg, V. G. Zakrzewski, S. Dapprich, A.D. Daniels, M. C. Strain, O. Farkas, D. K. Malick, A.D. Rabuck, K. Raghavachari, J. B. Foresman, J. V. Ortiz, Q. Cui, A. G. Baboul, S. Clifford, J. Cioslowski, B. B. Stefanov, G. Liu, A. Liashenko, P. Piskorz, I. Komaromi, R. L. Martin, D. J. Fox, T. Keith, M. A. Al-Laham, C. Y. Peng, A. Nanayakkara, M. Challacombe, P. M. W. Gill, B. Johnson, W. Chen, M. W. Wong, C. Gonzalez, J. A. Pople, GAUSSIAN 03 (revision C.02), Gaussian, Inc., Wallingford, CT (USA) 2004.

[19] J. Fabian, Chem. Rev. 1992, 92, 1197.

[20] A. J. Heeger, J. Phys. Chem. B 2001, 105, 8475.

[21] P. M. Lahti, ed., Magnetic Properties of Organic Materials, Marcel Dekker, New York, 1999.

[22] K. Itoh, M. Kinoshita, eds., Molecular Magnetism, Kodansha, Gordon and Beach, Tokyo, 2000. 